\title{
Metric fixed point theory for nonexpansive mappings defined on unbounded sets
}

\author{
Maryam A Alghamdi ${ }^{1}$, William A Kirk² and Naseer Shahzad ${ }^{3 *}$
}

${ }^{\text {"Correspondence: }}$

nshahzad@kau.edu.sa

${ }^{3}$ Department of Mathematics, King Abdulaziz University, P.O. Box 80203

Jeddah, 21589, Saudi Arabia

Full list of author information is

available at the end of the article

\begin{abstract}
It is standard practice in metric fixed point theory to reduce fixed point questions for mappings defined on unbounded sets to the bounded case. Many of these results are couched in a Banach space framework and involve bounded orbits. We examine these results in a somewhat broader metric context here.
\end{abstract}

MSC: Primary 54H25; 47H09

Keywords: nonexpansive mappings; fixed points; CAT(0) spaces

\section{Introduction}

If a closed convex subset has the fixed point property for all nonexpansive self-mappings, then is it necessarily bounded? This has long been an open question in metric fixed point theory. The answer is 'yes' if $X$ is a Hilbert space (see [1]). It has been shown recently (see [2]) that the failure of the fixed point property for every unbounded convex closed set is not a characteristic of Hilbert spaces; more precisely, for every unbounded closed convex set in $c_{0}$, there exists a fixed point free nonexpansive self-mapping of the set. On the other hand, it is obvious that nontrivial nonexpansive mappings defined on unbounded sets may have fixed points. Consider, for example, simple rotations in the plane. However, in this case the mapping has bounded orbits. Indeed, the following result is found in the original 1965 paper of Kirk [3].

Theorem 1.1 Suppose $K$ is a nonempty closed and convex subset of a reflexive Banach space, and suppose $K$ has a normal structure. Suppose $f: K \rightarrow K$ is a nonexpansive mapping, and suppose $\left\{f^{n}(p)\right\}$ is bounded for some (hence all) $p \in K$. Then $f$ has a fixed point.

The proof rests on the following fact (also proved in [3]).

Lemma 1.1 Suppose $K$ is a convex subset of a normed linear space and suppose $f: K \rightarrow K$ is nonexpansive. If $\left\{f^{n}(p)\right\}$ is bounded for some $p \in K$, then some bounded convex subset of $K$ is mapped into itself by $f$.

The above observations served as motivation for the following result.

Theorem 1.2 (Theorem 3.1 of [4]) Let $C$ be a closed convex subset of a Banach space $X$, let $\mathfrak{F}$ be a finite commuting family of nonexpansive self-mappings of $C$, and suppose $\left\{f^{n}(p)\right\}$ 
is bounded for some $p \in C$ and all $f \in \mathfrak{F}$. Then there is a nonempty bounded closed and convex subset of $C$ which is mapped into itself by each member of $\mathfrak{F}$.

This theorem in conjunction with Theorem 4 of [5] assures that in the setting of Theorem 1.1 finite commuting families of nonexpansive mappings with bounded orbits always have a common fixed point.

It is our objective in this paper to examine when analogs of the above results hold in broader contexts, and whether they hold for more general classes of mappings.

\section{The setting}

The results in this paper will depend strongly on the notions of metric convexity. The following definition is discussed in detail by Kohlenbach in [6].

$(X, \rho, W)$ is called a hyperbolic space if $(X, \rho)$ is a metric space and $W: X \times X \times[0,1] \rightarrow X$ is a function satisfying

(i) $\forall x, y, z \in X$ and $\forall \lambda \in[0,1]$,

$$
\rho(z, W(x, y, \lambda)) \leq(1-\lambda) \rho(z, x)+\lambda \rho(z, y)
$$

(ii) $\forall x, y \in X$ and $\forall \lambda_{1}, \lambda_{2} \in[0,1]$,

$$
\rho\left(W\left(x, y, \lambda_{1}\right), W\left(x, y, \lambda_{2}\right)\right)=\left|\lambda_{1}-\lambda_{2}\right| \rho(x, y)
$$

(iii) $\forall x, y \in X$ and $\forall \lambda \in[0,1], W(x, y, \lambda)=W(y, x, 1-\lambda)$;

(iv) $\forall x, y, z, w \in X$ and $\forall \lambda \in[0,1]$,

$$
\rho(W(x, z, \lambda), W(y, w, \lambda)) \leq(1-\lambda) \rho(x, y)+\lambda \rho(z, w) .
$$

If only condition (i) is satisfied, then $(X, \rho, W)$ is a convex metric space in the sense of Takahashi (cf. [7]). The first three conditions are equivalent to saying $(X, \rho, W)$ is a space of hyperbolic type in the sense of [8]. In this case the set

$$
[x, y]:=\{W(x, y, \lambda): \lambda \in[0,1]\}
$$

is called the metric segment joining $x$ and $y$ (condition (iii) ensures that $[x, y]$ is an isometric image of the real line interval $[0, \rho(x, y)])$. Hyperbolic spaces include all normed linear spaces and convex subsets thereof, as well as all CAT(0) spaces in the sense of Gromov (see [9]). Another important class of hyperbolic spaces are the so-called Busemann spaces (see [10]). These are precisely the hyperbolic spaces that are uniquely geodesic [11]. (We will not invoke condition (iv) in this paper.) For fixed point theory in these spaces, we refer the reader to [12-18].

We say that a subset $K$ of a Takahashi convex metric space is convex if $W(x, y, \lambda) \in K$ for all $x, y \in K$ and $\lambda \in[0,1]$. For some of our results discussed below this is all that is needed. With this convention all closed and open metric balls are convex and the intersection of any family of convex sets is also convex. We use $B(x ; r)$ to denote the closed ball centered at $x \in X$ with radius $r>0$. We adopt the customary notation and write $W(x, y, \lambda)=(1-$ $\lambda) x \oplus \lambda y$. 


\section{Preliminaries}

We begin with an abstract version of Lemma 1.1.

Lemma 3.1 Suppose $K$ is a convex subset of a Takahashi convex metric space and suppose $f: K \rightarrow K$ is nonexpansive. If $\left\{f^{n}(p)\right\}$ is bounded for some $p \in K$, then some bounded convex subset of $K$ is mapped into itself by $f$.

Proof Choose $r>0$ so that $\rho\left(p, f^{n}(p)\right) \leq r$ for each $n \in \mathbb{N}$, and let $K_{n}=B\left(f^{n}(p) ; r\right) \cap K$. If $u \in K_{n}$, then $\rho\left(u, f^{n}(p)\right) \leq r$; hence $\rho\left(f(u), f^{n+1}(p)\right) \leq \rho\left(u, f^{n}(p)\right) \leq r$, and it follows that $f(u) \in K_{n+1}$. For each $k \in \mathbb{N}$, let

$$
W_{k}=\bigcap_{i=k}^{\infty} K_{i}
$$

Then $f: W_{k} \rightarrow W_{k+1}$. Also $p \in W_{k}$ for each $k$, so $W_{k}$ is nonempty. Clearly $W_{k}$ is convex and bounded $\left(\operatorname{diam}\left(W_{k}\right) \leq 2 r\right)$. Therefore $\left\{W_{k}\right\}_{k=1}^{\infty}$ is an increasing sequence of uniformly bounded convex sets in $K$. It follows that $W=\bigcup_{k=0}^{\infty} W_{k}$ is a bounded convex set which is invariant under $f$.

A Takahashi convex metric space $X$ is said to have the FPP if every bounded closed convex subset of $X$ has the fixed point property for nonexpansive mappings. In view of Lemma 3.1 the following is immediate.

Theorem 3.1 Let $X$ be a Takahashi convex metric space which has the FPP, and let $K$ be a nonempty closed and convex subset of $X$. Suppose $f: K \rightarrow K$ is a nonexpansive mapping, and suppose $\left\{f^{n}(p)\right\}$ is bounded for some $p \in K$. Then $f$ has a fixed point.

Remark 1 In connection with Theorem 3.1 the following example is noteworthy.

Example If $K$ is an admissible subset (i.e., and intersection of closed balls) of a hyperconvex metric space $X$, then the set $W$ in the proof of Lemma 3.1 is the union of an increasing sequence of admissible sets. However, the closure of $W$ need not be admissible, or even hyperconvex. Stan Prus has given an example of a fixed point free nonexpansive mapping (actually an isometry) defined on $H=\ell_{\infty}$ which has bounded orbits. Indeed define $T: H \rightarrow H$ by setting

$$
T\left(\left(x_{1}, x_{2}, x_{3}, \ldots\right)\right)=\left(1+\lim _{\mathcal{U}} x_{n}, x_{1}, x_{2}, \ldots\right)
$$

where $\mathcal{U}$ is a nontrivial ultrafilter on the set of positive integers. The mapping $T$ is an isometry and has no fixed point. On the other hand, for $n \in \mathbb{N}$,

$$
T^{n}((0,0,0, \ldots))=(\overbrace{1,1, \ldots, 1}^{n \text {-times }}, 0,0, \ldots),
$$

so $T$ has bounded orbits.

\section{Eventually nonexpansive maps}

In this section we point out that Theorem 3.1 extends to a wider class of mappings in more restricted settings. 
Definition 4.1 Let $(X, \rho)$ be a metric space. A mapping $T: X \rightarrow X$ is said to be eventually uniformly Lipschitzian if there exist a sequence $\left\{k_{n}\right\}$ of positive numbers and an integer $N \in \mathbb{N}$ such that for all $n \geq N$,

$$
\rho\left(T^{n} x, T^{n} y\right) \leq k_{n} \rho(x, y)
$$

for all $x, y \in X$. If $\lim _{n \rightarrow \infty} k_{n}=1, T$ is said to be asymptotically nonexpansive. If $k_{n} \equiv 1$ for $n$ sufficiently large, $T$ is said to be eventually nonexpansive (see [19]). The following lemma is obtained by slightly adjusting the argument in the proof of Lemma 3.1.

Lemma 4.1 Suppose $K$ is a convex subset of a Takahashi convex metric space and suppose $f: K \rightarrow K$ is a mapping which is eventually nonexpansive. If $\left\{f^{n}(p)\right\}$ is bounded for some $p \in K$, then there exist $n_{0} \in N$ and a bounded convex subset of $K$ which is mapped into itself by each of the mappings $f^{n}, n \geq n_{0}$.

Proof Choose $r>0$ so that $\rho\left(p, f^{n}(p)\right) \leq r$ for each $n \in \mathbb{N}$, let $K_{n}=B\left(f^{n}(p) ; r\right) \cap K$, and for each $k \in \mathbb{N}$, let

$$
W_{k}=\bigcap_{i=k}^{\infty} K_{i} .
$$

Also $p \in W_{k}$ for each $k$, so $W_{k}$ is nonempty. Clearly $W_{k}$ is convex and bounded $\left(\operatorname{diam}\left(W_{k}\right) \leq 2 r\right)$. Therefore $\left\{W_{k}\right\}_{k=1}^{\infty}$ is an increasing sequence of uniformly bounded convex sets in $K$. It follows that $W=\bigcup_{k=0}^{\infty} W_{k}$ is bounded and convex.

Since $f$ is eventually nonexpansive, there exists $n_{0} \in \mathbb{N}$ such that $u \in K_{n} \Rightarrow \rho\left(f^{m}(u)\right.$, $\left.f^{n+m}(p)\right) \leq \rho\left(u, f^{n}(p)\right) \leq r$ for $m \geq n_{0}$. Thus $f^{m}(u) \in K_{n+m}$. So, for $m$ sufficiently large, $f^{m}$ : $K_{n} \rightarrow K_{n+m}$. In particular, $\bar{W}$ is a bounded closed convex subset of $K$ which is invariant under $f^{m}$.

Theorem 4.1 Let $X$ be a reflexive or separable Banach space which has the FPP, let $K$ be a closed convex subset of $X$, and suppose $f: K \rightarrow K$ is eventually nonexpansive. If $f^{n}(p)$ is bounded for some $p \in K$, then $f$ has a fixed point.

Proof Let $W$ be as in Lemma 4.1. Then in particular $\bar{W}$ is a bounded closed convex set which in invariant under the commuting nonexpansive mappings $f^{m}$ and $f^{m+1}$. One can now apply a classical result of Bruck [20] to conclude that $f^{m}$ and $f^{m+1}$ have a common fixed point which is necessarily a fixed point of $f$.

$\mathbb{R}$-trees (or metric trees) are a class of hyperbolic spaces which have interesting geometric properties.

Definition 4.2 An $\mathbb{R}$-tree is a metric space $X$ such that

(i) there is a unique geodesic (metric) segment denoted by $[x, y]$ joining each pair of points $x$ and $y$ in $X$; and

(ii) $[y, x] \cap[x, z]=\{x\} \Rightarrow[y, x] \cup[x, z]=[y, z]$.

Theorem 4.1 extends to complete $\mathbb{R}$-trees without any additional assumptions. 
Theorem 4.2 Let $(X, \rho)$ be a complete $\mathbb{R}$-tree, let $K$ be a closed convex subset of $X$, and suppose $f: K \rightarrow K$ is a mapping which is eventually nonexpansive and for which $f^{n}(p)$ is bounded for some $p \in K$. Then $f$ has a fixed point.

Theorem 4.2 is an immediate consequence of the following two facts. Proposition 4.1 was first proved in [21]. For convenience of the reader, we repeat the proof here.

Theorem 4.3 ([22]) Let $(X, \rho)$ be a complete $\mathbb{R}$-tree, and suppose $T: X \rightarrow X$ has bounded orbits and satisfies, for all $n \in \mathbb{N}$ sufficiently large,

$$
\rho\left(T^{n} x, T^{n} y\right) \leq k_{n} \rho(x, y)
$$

for all $x, y \in X$, where $\limsup _{n \rightarrow \infty} k_{n}<2$. Then $T$ has a fixed point.

Proposition 4.1 Let $(X, \rho)$ be a metric space and suppose $T: X \rightarrow X$ is eventually uniformly Lipschitzian for a sequence $\left\{k_{n}\right\}$, and suppose $T$ has a bounded orbit. If $\limsup _{n \rightarrow \infty} k_{n}<\infty$, then all orbits of $T$ are bounded.

Proof Assume there exist $x \in X$ and $r>0$ such that $\left\{T^{n}(x)\right\} \subset B(x ; r)$. Choose $k>0$ so that $\lim \sup _{n \rightarrow \infty} k_{n}<k$. Then, if $y \in X$, it is possible to choose $m \in \mathbb{N}$ so that for all $n \geq m$,

$$
\rho\left(T^{n}(x), T^{n}(y)\right) \leq k \rho(x, y) .
$$

Then, for $n \geq m$,

$$
\rho\left(x, T^{n}(y)\right) \leq \rho\left(x, T^{n}(x)\right)+\rho\left(T^{n}(x), T^{n}(y)\right) \leq r+k \rho(x, y) .
$$

This proves that $\left\{T^{n}(y)\right\}_{n \geq m} \subset B(x ; d)$, where $d=r+k \rho(x, y)$. Let

$$
d^{\prime}=\max \left\{\rho\left(x, T^{i}(y)\right): i=1, \ldots, m-1\right\} .
$$

Then $\left\{T^{n}(y)\right\} \subset B\left(x ; d^{*}\right)$, where $d^{*}=\max \left\{d, d^{\prime}\right\}$. Since $y$ is arbitrary, all orbits of $T$ are bounded.

Remark Some form of asymptotic control over the behavior of the mapping is needed for the validity of Proposition 4.1, even if the mapping is continuous and $X$ is the real line. It is easy to construct continuous mappings of the real line that have exactly one fixed point and all other orbits are unbounded. However, it is shown in [21] that if $T$ is assumed to be continuous in Theorem 4.3, then the assumption that $\lim _{\sup _{n \rightarrow \infty}} k_{n}<2$ may be replaced with the much weaker assumption that $\lim _{\sup _{n \rightarrow \infty}} k_{n}<\infty$.

\section{Bounded orbits of families of mappings}

Our next theorem is an analog of Theorem 3.1 in [4] which is formulated there in a Banach space setting. Note that commutativity of $\mathfrak{F}$ appears (at least in some sense) to be essential to the proof. As noted in [4], this result shows that the assumption of strict convexity is not needed for Theorem 4 of [23]. (As Bula remarks in [23], this theorem is not true for infinite families.) 
Theorem 5.1 Let $(X, \rho)$ be a Takahashi convex metric space, and let $K$ be a convex subset of $X$, and let $\mathfrak{F}$ be a finite commutative family of nonexpansive self-mappings of $K$. Suppose $\left\{f^{n}(p)\right\}$ is bounded for some (and hence all) $p \in K$ and each $f \in \mathfrak{F}$. Then there is a bounded convex subset of $K$ which is left invariant by each member of $\mathfrak{F}$.

Proof We first prove the theorem when $\mathfrak{F}=\{f, g\}$. The general case is a straightforward adaptation of this procedure (although the details are rather tedious). By assumption there exist $r_{1}, r_{2}>0$ such that $\left\{f^{n}(p)\right\} \subset B\left(p ; r_{1}\right)$ and $\left\{g^{n}(p)\right\} \subset B\left(p ; r_{2}\right)$. Thus, for $m, n \in \mathbb{N}$,

$$
\begin{aligned}
\rho\left(f^{n} \circ g^{m}(p), p\right) & \leq \rho\left(f^{n} \circ g^{m}(p), f^{n}(p)\right)+\rho\left(f^{n}(p), p\right) \\
& \leq \rho\left(g^{m}(p), p\right)+\rho\left(f^{n}(p), p\right) \\
& \leq r_{2}+r_{1}:=r .
\end{aligned}
$$

Therefore $\left\{f^{n} \circ g^{m}(p)\right\}_{m, n=1}^{\infty} \subset B(p ; r)$. For each $m, n \in \mathbb{N}$, let

$$
\begin{aligned}
S_{n, m} & :=\left\{u \in K: \rho\left(u, f^{i} \circ g^{j}(p)\right) \leq r \forall i \geq n, j \geq m\right\} \\
& =\left(\bigcap_{i \geq n, j \geq m} B\left(f^{i} \circ g^{j}(p) ; r\right)\right) \cap K,
\end{aligned}
$$

and let $S=\bigcup_{n, m=1}^{\infty} S_{n, m}$. Because balls in $X$ are convex, each of the sets $S_{m, n}$ is convex. Moreover, the family $\left\{S_{m, n}\right\}_{m, n=1}^{\infty}$ is directed upward by set inclusion, so $S$ is convex. Also, if $u \in S_{n, m}$, then

$$
\rho\left(f(u), f^{i} \circ g^{j}(u)\right) \leq \rho\left(u, f^{i-1} \circ g^{j}(u)\right) \leq r \quad \forall i-1 \geq n, j \geq m,
$$

so $f(u) \in S_{n+1, m}$. Similarly,

$$
\begin{aligned}
\rho\left(g(u), f^{i} \circ g^{j}(u)\right) & =\rho\left(g(u), g \circ f^{i} \circ g^{j-1}(u)\right) \\
& \leq \rho\left(u, f^{i} \circ g^{j-1}(u)\right) \leq r \quad \forall i \geq n, j-1 \geq m
\end{aligned}
$$

and $g(u) \in S_{n, m+1}$. (Notice that here we use the fact that the mappings $f$ and $g$ commute.) It follows that $S$ is a bounded convex set which is invariant under both $f$ and $g$.

We now briefly indicate how to prove Theorem $5.1 \mathrm{in}$ its full generality. (The assertion in [4] that the general case follows by induction seems to oversimplify the situation.) Suppose $\mathfrak{F}=\left\{f_{1}, \ldots, f_{k}\right\}$ and let

$$
r_{0}=\sup \left\{\rho\left(p, f_{j}^{n}(p)\right): 1 \leq j \leq k, n=1,2, \ldots\right\} .
$$

Then

$$
\begin{aligned}
& \rho\left(p, f_{1}^{i_{1}} \circ f_{2}^{i_{2}} \circ \cdots \circ f_{k}^{i_{k}}(p)\right) \\
& \quad \leq \rho\left(p, f_{1}^{i_{1}}(p)\right)+\rho\left(f_{1}^{i_{1}}(p), f_{1}^{i_{1}} \circ f_{2}^{i_{2}} \circ \cdots \circ f_{k}^{i_{k}}(p)\right) \\
& \quad \leq r_{0}+\rho\left(p, f_{2}^{i_{2}} \circ \cdots \circ f_{k}^{i_{k}}(p)\right) \\
& \quad \leq r_{0}+\rho\left(p, f_{2}^{i_{2}}(p)\right)+\rho\left(f_{2}^{i_{2}}(p), f_{2}^{i_{2}} \circ \cdots \circ f_{k}^{i_{k}}(p)\right)
\end{aligned}
$$




$$
\begin{aligned}
& \leq 2 r_{0}+\rho\left(p, f_{3}^{i_{3}} \circ \cdots \circ f_{k}^{i_{k}}(p)\right) \\
& \leq \cdots \\
& \leq k r_{0}:=r .
\end{aligned}
$$

Let

$$
S\left(i_{1}, i_{2}, \ldots, i_{k}\right)=\bigcap_{m_{j} \geq i_{j}} B\left(f_{1}^{m_{1}} \circ f_{2}^{m_{2}} \circ \cdots \circ f_{k}^{m_{k}}(p) ; r\right) \cap K .
$$

Then $p$ is in each of the sets $S\left(i_{1}, i_{2}, \ldots, i_{k}\right)$. The family

$$
\left\{S\left(i_{1}, i_{2}, \ldots, i_{k}\right)\right\}
$$

of convex sets is directed upward by set inclusion because any two sets

$$
S\left(i_{1}, i_{2}, \ldots, i_{k}\right) \text { and } S\left(j_{1}, j_{2}, \ldots, j_{k}\right)
$$

are contained in $S\left(n_{1}, n_{2}, \ldots, n_{k}\right)$, where $n_{v}=\max \left(i_{v}, j_{v}\right), v=1, \ldots, k$. Let

$$
S=\bigcup_{i_{1}, \ldots, i_{k}=1}^{\infty} S\left(i_{1}, i_{2}, \ldots, i_{k}\right) .
$$

Then $S$ is a bounded convex set which is invariant under each of the mappings in $\mathfrak{F}$.

Theorem 5.1 has a different proof if the space $X$ is of hyperbolic type. For this we need the following fact. Recall that a mapping $f$ of a metric space $(X, \rho)$ into itself is said to be asymptotically regular if for each $x \in X, \lim _{n \rightarrow \infty} \rho\left(f^{n}(x), f^{n+1}(x)\right)=0$. The following is a consequence of results of [8]; also see [24].

Proposition 5.1 Let $K$ be a bounded convex subset of a space $(X, \rho)$ of hyperbolic type, and suppose $f: K \rightarrow K$ is nonexpansive. Fix $\alpha \in(0,1)$, and define $f_{\alpha}: K \rightarrow K$ by setting $f_{\alpha}(x)=$ $\alpha x \oplus(1-\alpha) f(x)$. Then $f_{\alpha}$ is asymptotically regular. In particular, $\inf \{\rho(x, f(x)): x \in K\}=0$.

Second proof of Theorem 5.1 We consider only the case $\mathfrak{F}=\{f, g\}$. If $X$ is of hyperbolic type, in view of Lemma 3.1 some bounded convex subset $H$ of $K$ is mapped into itself by $g$. Consequently, by Proposition 5.1

$$
\inf \{\rho(x, g(x)): x \in H\}=0 .
$$

Let $\delta>0$ and choose $p \in H$ such that $\rho(p, g(p)) \leq \delta$. Let

$$
F_{\delta}(g):=\{x \in K: \rho(x, g(x)) \leq \delta\}
$$

Clearly $g: F_{\delta}(g) \rightarrow F_{\delta}(g)$. Let $x \in F_{\delta}(g)$. Then

$$
\rho(g \circ f(x), f(x))=\rho(f \circ g(x), f(x)) \leq \rho(g(x), x) \leq \delta .
$$


Therefore it is also the case that $f: F_{\delta}(g) \rightarrow F_{\delta}(g)$, and thus $f^{i} \circ g^{j}(p)=g \circ f^{i} \circ g^{j-1}(p)$ for all $i, j \geq 0$.

The proof is now completed as in the first proof.

Remark 2 An interesting feature of the second proof is that it is only necessary to assume that $f$ and $g$ commute on the set $F_{\delta}(g)$ for some $\delta>0$ rather than on the entire domain.

\section{A condition of Djebali-Hammache}

In [25] the authors present some new versions of fixed point theorems for nonexpansive mappings defined on closed, convex subsets of Banach spaces which are not necessarily bounded. In this section we discuss a result which they compare with Theorem 2.4 of [4] (see below). The following definition and notation are taken from [25].

Definition 6.1 Let $Q$ be a nonempty closed convex subset of a Banach space $X$. A mapping $f: Q \rightarrow X$ is said to have the property $(\mathcal{K})$ if there exists a nonempty bounded closed convex subset $K \subset X$ such that $f(Q \cap K) \subset K$.

(Implicit in the above is the assumption also that $Q \cap K \neq \emptyset$.)

Notation 1 Define the set

$$
S=S(f, Q)=\left\{\left\{x_{n}\right\} \subset Q: x_{n}=\left(1-\frac{1}{n}\right) f\left(x_{n}\right) \forall n \in \mathbb{N}\right\} .
$$

By the Banach contraction mapping theorem, this set is always nonempty if $f$ is nonexpansive and $Q$ is a nonempty convex subset of $X$ which contains the origin.

Now let $A \subset X$ be nonempty and bounded, and let $\alpha(A)$ denote the Kuratowski measure of noncompactness of $A$. For $\varepsilon, c>0$ with $0<c<\alpha(A)+\varepsilon$, set

$$
N_{\varepsilon}^{c}(A)=\left\{(x, y) \in A^{2}: c \leq\|x-y\| \leq \alpha(A)+\varepsilon\right\} .
$$

This set is denoted by $N_{\varepsilon}^{c}(f, A)$ when $A$ depends explicitly on some function $f$.

Let $S$ be given by (6.1), and for any closed bounded convex subset $K$ of $X$, define

$$
S_{K}=S \cap K
$$

The following is Theorem 3.2 of [25]. Here

$$
F_{\delta_{0}}\left(f, S_{K}\right)=\left\{x \in S_{K}:\|x-f(x)\| \leq \delta_{0}\right\}
$$

Theorem 6.1 Let $X$ be a Banach space, $Q$ be a convex closed subset of $X$ containing the origin, and let $f: Q \rightarrow Q$ be a nonexpansive mapping satisfying property $(\mathcal{K})$. Let $K$ be the bounded closed convex subset of $X$ whose existence is assured by Definition 6.1. Assume that there exist $\delta_{0}, \varepsilon_{0}>0$ such that $\forall c \in\left(0, \alpha\left(S_{K}\right)+\varepsilon_{0}\right)$,

$$
\left[F_{\delta_{0}}\left(f, S_{K}\right) \times F_{\delta_{0}}\left(f, S_{K}\right)\right] \cap N_{\varepsilon_{0}}^{c}\left(f, S_{K}\right)=\emptyset .
$$

Then $f$ has a fixed point. 
This theorem is an immediate consequence of the following lemma (Lemma 3.1 in [25]).

Lemma 6.1 Under the assumptions of Theorem 6.1, $\alpha\left(S_{K}\right)=0$.

A Banach space $X$ is said to have the FPP if each of its bounded closed convex subsets has the fixed point property for nonexpansive self-mappings. The following is Theorem 2.4 of [4].

Theorem 6.2 Let $X$ be a Banach space which has the FPP, let $C$ be a closed convex subset of $X$, and suppose $f: C \rightarrow C$ is a nonexpansive mapping for which $F_{\delta}(f, C)$ is nonempty and bounded for some $\delta>0$. Then $f$ has a fixed point.

The authors of [25] compare Theorem 6.1 with Theorem 6.2 and remark that in Theorem 6.1 the boundedness of $F_{\delta}(f, Q)$ is relaxed and the assumption that the space has the FPP is dropped. However, the added condition in Theorem 6.1 that the mapping satisfies property $(\mathcal{K})$ in conjunction with the fact that $f: Q \rightarrow Q$ implies $f: Q \cap K \rightarrow Q \cap K$ immediately reduces Theorem 6.1 to the bounded case. Also, the nonexpansiveness of $f$ is used in the proof of Theorem 6.1 only to guarantee the existence of an approximate fixed point sequence for $f$ and to guarantee that $f$ is continuous. Finally, condition (6.2) in Theorem 6.1 is deceptively strong and, as the following result shows, Theorem 6.1 is essentially trivial.

Theorem 6.3 Let $X$ be a Banach space, $Q$ be a subset of $X$, let $f: Q \rightarrow Q$ be a mapping, and suppose $f$ has a bounded approximate fixed point sequence $S$ in $Q$. Also assume that there exist $\delta_{0}, \varepsilon_{0}>0$ such that $\forall c \in\left(0, \alpha(S)+\varepsilon_{0}\right)$,

$$
\left[F_{\delta_{0}}(f, S) \times F_{\delta_{0}}(f, S)\right] \cap N_{\varepsilon_{0}}^{c}(f, S)=\emptyset .
$$

Then $S$ is finite. (Thus, $f$ can have no nontrivial approximate fixed point sequence.)

The proof of this theorem hinges on the fact that since $c \in\left(0, \alpha(S)+\varepsilon_{0}\right)$ is arbitrary, condition (6.3) actually implies the following. There exist $\delta_{0}, \varepsilon_{0}>0$ such that for $x, y \in S$, if $\|x-f(x)\| \leq \delta_{0}$ and $\|y-f(y)\| \leq \delta_{0}$, then $\|x-y\|<\alpha(S)+\varepsilon_{0} \Rightarrow x=y$. Otherwise one could take $c=\|x-y\|$ and conclude that

$$
\left[F_{\delta_{0}}(f, S) \times F_{\delta_{0}}(f, S)\right] \cap N_{\varepsilon_{0}}^{c}(f, S) \neq \emptyset .
$$

We omit the details since a more general theorem is proved below.

There is a weaker version of condition (6.3) that is somewhat more realistic. In fact this appears to be the version the authors of [25] actually use in their applications.

In the following $(X, \rho)$ denotes a metric space, $Q \subset X$ and $f: Q \rightarrow Q$. For $\delta>0, F_{\delta}(f, S)$ and for $\varepsilon>0$ and $c \in(0, \alpha(S)+\varepsilon), N_{\varepsilon}^{c}(f, S)$ denote their Banach space analogs defined above with $\rho(\cdot)$ replacing $\|\cdot\|$.

Theorem 6.4 Let $Q$ be a closed subset of a complete metric space $(X, \rho)$, and suppose $f$ : $Q \rightarrow Q$ is a mapping which has a bounded approximate fixed point sequence $S$. Assume 
that there exists $\varepsilon>0$ such that $\forall c \in(0, \alpha(S)+\varepsilon)$ there exists $\delta>0$ such that

$$
\left[F_{\delta}(f, S) \times F_{\delta}(f, S)\right] \cap N_{\varepsilon}^{c}(f, S)=\emptyset .
$$

Then $\alpha(S)=0$. In particular, iff is continuous, then $f$ has a fixed point.

Proof Let $S:=\left\{x_{n}\right\}$ and assume $\alpha(S)>0$. Then, by passing to a subsequence if necessary, we may further assume that there exists $c>0$ such that for $x, y \in S, x \neq y \Rightarrow \rho(x, y) \geq c$. Also, by the definition of $\alpha$, there exist subsets of $\left\{\Omega_{i}\right\}_{i=1}^{m}$ of $S$ such that $S \subset \bigcup_{i=1}^{m} \Omega_{i}$ and such that for each $i \in\{1, \ldots, m\}, \operatorname{diam}\left(\Omega_{i}\right) \leq \alpha(S)+\varepsilon$. Therefore, for $x, y \in \Omega_{i}$,

$$
x \neq y \quad \Rightarrow \quad c \leq \rho(x, y) \leq \alpha(S)+\varepsilon
$$

thus $(x, y) \in N_{\varepsilon}^{c}(S)$. Now choose $i \in\{1, \ldots, m\}$ such that $\Omega_{i}$ is infinite. Then $\Omega_{i}$ contains an infinite number of terms of $\left\{x_{n}\right\}$ which lie in $F_{\delta}(f, S)$. In particular it is possible to choose $x, y \in \Omega_{i}$ such that $x \neq y$ and such that $(x, y) \in\left[F_{\delta}(f, S) \times F_{\delta}(f, S)\right]$. Therefore

$$
(x, y) \in\left[F_{\delta}(f, S) \times F_{\delta}(f, S)\right] \cap N_{\varepsilon}^{c}(S),
$$

which contradicts (6.4).

Remark In view of Theorem 6.4, condition (6.4) reduces to the following assumption. Given $c \in(0, \varepsilon)$ there exists $\delta(c)>0$ such that

$$
\left.\begin{array}{l}
\rho(x, f(x)) \leq \delta(c), \\
\rho(y, f(y)) \leq \delta(c)
\end{array}\right\} \Rightarrow \rho(x, y)<c \quad \text { or } \quad \rho(x, y)>\varepsilon .
$$

Now suppose $u$ and $v$ are fixed points of $f$ with $u \neq v$. Choose $c \in(0, \varepsilon)$ so that $c<\rho(u, v)$. Since $\rho(u, f(u)) \leq \delta(c)$ and $\rho(v, f(v)) \leq \delta(c)$, it must be the case that $\rho(u, v)>\varepsilon$. Thus condition (6.4) implies that the fixed point set of $f$ is always discrete.

We conclude with an application of Theorem 6.4 to mappings that are not necessarily continuous. Recall that the mapping $f: Q \rightarrow Q$ is said to satisfy Suzuki's condition (C) on $Q$ if

$$
\frac{1}{2} \rho(x, f(x)) \leq \rho(x, y) \quad \Rightarrow \quad \rho(f(x), f(y)) \leq \rho(x, y)
$$

for all $x, y \in Q$ (see [26]).

Theorem 6.5 Let $Q$ be a bounded closed subset of a complete metric space $(X, \rho)$, and suppose $f: Q \rightarrow Q$ is a mapping which has a bounded approximate fixed point sequence $S$. Assume that there exists $\varepsilon>0$ such that $\forall c \in(0, \alpha(S)+\varepsilon)$ there exists $\delta>0$ such that

$$
\left[F_{\delta}(f, S) \times F_{\delta}(f, S)\right] \cap N_{\varepsilon}^{c}(f, S)=\emptyset .
$$

Iff satisfies Suzuki's condition (C), then $f$ has a fixed point. 
Proof We follow the argument used in Theorem 2 of [26]. It follows from Theorem 6.4 that $\alpha(S)=0$. There exists a subsequence $\left\{x_{n}\right\}$ of $S$ and $z \in Q$ such that $\left\{x_{n}\right\}$ converges to $z$. By Lemma 7 of [26] (which can be formulated for metric spaces), we have

$$
\rho\left(x_{n}, f(z)\right) \leq 3 \rho\left(x_{n}, f\left(x_{n}\right)\right)+\rho\left(x_{n}, z\right)
$$

for all $n$. So $\left\{x_{n}\right\}$ converges to $f(z)$ and hence $f(z)=z$.

Remark If $Q$ is a bounded convex subset of a Banach space and $f$ satisfies Suzuki's condition $(\mathrm{C})$, then $f$ always has a bounded approximate fixed point sequence by Lemma 6 of [26].

\section{Historical comment about bounded orbits}

A G-space $R$ in the sense of Busemann [27] is a metric space which is (i) finitely compact (or proper, i.e., bounded closed sets are compact), (ii) metrically convex, and for which (iii) prolongation is locally possible and unique. Precisely, (iii) means that to every point $p \in R$ there corresponds a number $\rho_{p}>0$ such that if $x, y \in U\left(p ; \rho_{p}\right)$ (the open ball) with $x \neq y$, there exists a point $z \in R$ for which

$$
d(x, y)+d(y, z)=d(x, z)
$$

and moreover, if $d(x, y)+d\left(y, z_{1}\right)=d\left(x, z_{1}\right)$ and $d(x, y)+d\left(y, z_{2}\right)=d\left(x, z_{2}\right)$, then $d\left(y, z_{1}\right)=$ $d\left(y, z_{2}\right) \Rightarrow z_{1}=z_{2}$.

Theorem 7.1 ([28]) If $R$ is a straight G-space (has unique metric segments) which has convex spheres, and if $\phi$ is a motion of $R$ (an isometry of $R$ onto itself) for which $\left\{\phi^{n}(p)\right\}$ is bounded for some $p \in R$, then $\phi$ has a fixed point.

It was subsequently shown in Kirk [29] that it suffices to assume only that some subsequence of $\left\{\phi^{n}(p)\right\}$ is bounded in Theorem 7.1, an assumption later shown by Całka [30] to be (nontrivially) equivalent to the original.

Competing interests

The authors declare that they have no competing interests.

Authors' contributions

All authors contributed equally and significantly in writing this paper. All authors read and approved the final manuscript.

\section{Author details}

${ }^{1}$ Faculty of Science for Girls, King Abdulaziz University, P.O. Box 4087, Jeddah, 21491, Saudi Arabia. ${ }^{2}$ Department of Mathematics, University of lowa, lowa City, lowa 42242, USA. ${ }^{3}$ Department of Mathematics, King Abdulaziz University, P.O. Box 80203, Jeddah, 21589, Saudi Arabia.

\section{Acknowledgements}

This article was funded by the Deanship of Scientific Research (DSR), King Abdulaziz University, Jeddah. The authors, therefore, acknowledge with thanks DSR for financial support. The authors are grateful to anonymous reviewers for useful comments.

Received: 26 March 2014 Accepted: 1 July 2014 Published: 22 July 2014

\section{References}

1. Ray, WO: The fixed point property and unbounded sets in Hilbert space. Trans. Am. Math. Soc. 258(2), $531-537$ (1980)

2. Domínguez Benavides, T: The failure of the fixed point property for unbounded sets in $c_{0}$. Proc. Am. Math. Soc. 140, 645-650 (2012) 
3. Kirk, WA: A fixed point theorem for mappings which do not increase distances. Am. Math. Mon. 72, 1004-1006 (1965)

4. Kaewcharoen, A, Kirk, WA: Nonexpansive mappings defined on unbounded domains. Fixed Point Theory Appl. 2006, Article ID 82080 (2006)

5. Belluce, LP, Kirk, WA: Fixed-point theorems for families of contraction mappings. Pac. J. Math. 18, $213-217$ (1966)

6. Kohlenbach, U: Some logical metatheorems with applications in functional analysis. Trans. Am. Math. Soc. 357(1), 89-128 (2005) (electronic)

7. Takahashi, W: A convexity in metric space and nonexpansive mappings. I. Kodai Math. Semin. Rep. 22, 142-149 (1970)

8. Goebel, K, Kirk, WA: Iteration processes for nonexpansive mappings. In: Topological Methods in Nonlinear Functional Analysis (Toronto, Ont., 1982). Contemp. Math., vol. 21, pp. 115-123. Am. Math. Soc., Providence (1983)

9. Bridson, M, Haefliger, A: Metric Spaces of Non-positive Curvature. Grundlehren der Mathematischen Wissenschaften (Fundamental Principles of Mathematical Sciences), vol. 319. Springer, Berlin (1999)

10. Papadopoulos, A: Metric Spaces, Convexity and Nonpositive Curvature. IRMA Lectures in Mathematics and Theoretical Physics, vol. 6. Eur. Math. Soc., Zürich (2005)

11. Ariza-Ruiz, D, Leuştean, L, Lopez-Acedo, G: Firmly nonexpansive mappings in classes of geodesic spaces (2012). arXiv:1203.1432v3 [math.FA]

12. Alghamdi, MA, Kirk, WA, Shahzad, N: Remarks on convex combinations in geodesic spaces. J. Nonlinear Convex Anal. 15, 49-59 (2014)

13. Alghamdi, MA, Kirk, WA, Shahzad, N: Locally nonexpansive mappings in geodesic and length spaces. Topol. Appl. 173, 59-73 (2014)

14. Kirk, WA: Geodesic geometry and fixed point theory. In: Seminar of Mathematical Analysis (Malaga/Seville, 2002/2003). Colecc. Abierta, vol. 64, pp. 195-225. Univ. Sevilla Secr. Publ., Seville (2003)

15. Kirk, WA: Geodesic geometry and fixed point theory. II. In: International Conference on Fixed Point Theory and Applications, pp. 113-142. Yokohama Publ., Yokohama (2004)

16. Shahzad, N: Invariant approximations in CAT(0) spaces. Nonlinear Anal. 70, 4338-4340 (2009)

17. Shahzad, N: Fixed point results for multimaps in CAT(0) spaces. Topol. Appl. 156, 997-1001 (2009)

18. Shahzad, N, Markin, J: Invariant approximations for commuting mappings in CAT(0) and hyperconvex spaces. J. Math Anal. Appl. 337, 1457-1464 (2008)

19. Kirk, WA: The fixed point property and mappings which are eventually nonexpansive. In: Theory and Applications of Nonlinear Operators of Accretive and Monotone Type. Lecture Notes in Pure and Appl. Math., vol. 178, pp. 141-147. Dekker, New York (1996)

20. Bruck, RE: A common fixed point theorem for a commuting family of nonexpansive mappings. Pac. J. Math. 53, 59-71 (1974)

21. Kirk, WA, Shahzad, N: Uniformly Lipschitzian mappings in $\mathbb{R}$-trees (submitted)

22. Aksoy, AG, Khamsi, MA: Fixed points of uniformly Lipschitzian mappings in metric trees. Sci. Math. Jpn. 65, 31-41 (2007)

23. Bula, I: Some generalizations of W.A. Kirk's fixed point theorems. In: Mathematics. Latv. Univ. Zināt. Raksti, vol. 595, pp. 159-166. Latv. Univ., Riga (1994)

24. Kirk, WA: Krasnoselskii's iteration process in hyperbolic space. Numer. Funct. Anal. Optim. 4, 371-381 (1981/82)

25. Djebali, S, Hammache, K: Fixed point theorems for nonexpansive maps in Banach spaces. Nonlinear Anal. 73, 3440-3449 (2010)

26. Suzuki, T: Fixed point theorems and convergence theorems for some generalized nonexpansive mappings. J. Math Anal. Appl. 340(2), 1088-1095 (2008) (English summary)

27. Busemann, H: The Geometry of Geodesics. Academic Press, New York (1955)

28. Kirk, WA: Isometries in G-spaces. Duke Math. J. 31, 539-541 (1964)

29. Kirk, WA: On conditions under which local isometries are motions. Colloq. Math. 22, 229-232 (1971)

30. Całka, A: On conditions under which isometries have bounded orbits. Colloq. Math. 48(2), $219-227$ (1984)

doi:10.1186/1687-1812-2014-143

Cite this article as: Alghamdi et al.: Metric fixed point theory for nonexpansive mappings defined on unbounded sets. Fixed Point Theory and Applications 2014 2014:143.

\section{Submit your manuscript to a SpringerOpen ${ }^{\circ}$ journal and benefit from:}

- Convenient online submission

Rigorous peer review

- Immediate publication on acceptance

- Open access: articles freely available online

- High visibility within the field

- Retaining the copyright to your article 